\title{
Zebrafish Crip2 Plays a Critical Role in Atrioventricular Valve Development by Downregulating the Expression of ECM Genes in the Endocardial Cushion
}

\author{
Jun-Dae Kim ${ }^{1,4,5}$, Hey-Jin Kim ${ }^{1,5}$, Soonil Koun ${ }^{1}$, Hyung-Jin Ham ${ }^{1}$, Myoung-Jin Kim ${ }^{1}$, \\ Myungchull Rhee ${ }^{2}$, and Tae-Lin Huh ${ }^{1,3, *}$
}

\begin{abstract}
The initial step of atrioventricular (AV) valve development involves the deposition of extracellular matrix (ECM) components of the endocardial cushion and the endocardialmesenchymal transition. While the appropriately regulated expression of the major ECM components, Versican and Hyaluronan, that form the endocardial cushion is important for heart valve development, the underlying mechanism that regulates ECM gene expression remains unclear. We found that zebrafish crip2 expression is restricted to a subset of cells in the AV canal (AVC) endocardium at 55 hours post-fertilization (hpf). Knockdown of crip2 induced a heart-looping defect in zebrafish embryos, although the development of cardiac chambers appeared to be normal. In the AVC of Crip2-deficient embryos, the expression of both versican $a$ and hyaluronan synthase 2 (has2) was highly upregulated, but the expression of bone morphogenetic protein 4 (bmp4) and T-box $2 b(t b \times 2 b)$ in the myocardium and of notch1b in the endocardium in the AVC did not change. Taken together, these results indicate that crip2 plays an important role in AV valve development by downregulating the expression of ECM components in the endocardial cushion.
\end{abstract}

\section{INTRODUCTION}

The developing vertebrate heart tube contains an outer layer of

\begin{abstract}
${ }^{1}$ School of Life Science and Biotechnology (Brain Korea 21 plus program), Kyungpook National University, Daegu 702-701, Korea, ${ }^{2}$ Department of Biological Sciences, College of Bioscience and Biotechnology, Chungnam National University, Daejeon 305-764, Korea, ${ }^{3}$ Korea Basic Science Institute Daegu Center, Daegu 702-701, Korea, ${ }^{4}$ Present address: Yale Cardiovascular Research Center, Section of Cardiovascular Medicine, Department of Internal Medicine, Yale University School of Medicine, New Haven, CT, USA, ${ }^{5}$ These authors contributed equally to this work.

${ }^{*}$ Correspondence: thuh @knu.ac.kr
\end{abstract}

Received 27 March, 2014; revised 18 April, 2014; accepted 28 April, 2014; published online 14 May, 2014

Keywords: cardiac valve, crip2, has2, versican, zebrafish myocardium and an inner layer of endocardium separated by an extracellular matrix (ECM) referred to as the cardiac jelly. During heart valve formation, a subset of endocardial cells in the atrioventricular (AV) canal (AVC) region is specified to delaminate, differentiate, and migrate into the cardiac jelly in a process called endocardial-mesenchymal transition (EMT) (Armstrong and Bischoff, 2004; Combs and Yutzey, 2009). For AV valve formation, locally swollen cardiac jelly together with mesenchymal cells derived from AVC endocardial cells form the endocardial cushion; however the extensive remodeling process that occurs subsequently is not clearly understood (Armstrong and Bischoff, 2004; Combs and Yutzey, 2009).

Signal molecules produced from both the myocardium and endocardium in the AVC region are necessary for proper endocardial cushion formation and EMT of endocardial cells (Krug et al., 1985). In the initial step of endocardial cushion formation, a bone morphogenetic protein (BMP) signal from AVC myocardium inhibits the expression of chamber-specific genes in the AVC region, while the endocardial cushion is enlarged by increased deposition of ECM or cardiac jelly between the myocardium and endocardium layers in the AVC. Upon receiving a myocardially derived BMP signal, a subset of AVC endocardium cells undergo the EMT process by producing multiple signals such as transforming growth factor $\beta$, Notch, and Wnt $/ \beta$ catenin, and then migrate into the cardiac jelly where they transition into mesenchymal cells that form valve precursors during later development (Armstrong and Bischoff, 2004; Combs and Yutzey, 2009; Délot, 2003; Nakajima et al., 2000). Therefore, deficiencies in BMP or Notch signal-related genes inhibit or disrupt normal endocardial cushion formation and/or failure of AVC endocardium EMT in the mouse, chick, and zebrafish (Jiao et al., 2003; Ma et al., 2005; Timmerman et al., 2004). In addition, FGFs, VEGFs, NFAT, and HDAC are also prerequisites for normal heart valve development (Combs and Yutzey, 2009; Kim et al., 2012b).

Glycosaminoglycan hyaluronan exists as a hydrated gel that expands the extracellular space, regulates ligand availability, and interacts with numerous ECM components including versican, a major constituent of cardiac jelly (Armstrong and Bischoff, 2004). Disruption of hyaluronan synthase 2 (has2) or versican in mice and zebrafish is reported to abrogate AVC endocardial 
cushion formation and EMT-associated mesenchymal cell migration, suggesting that an appropriate ECM environment is important for both EMT of AVC endocardium development and formation of the endocardial cushion (Camenisch et al., 2000; Mjaatvedt et al., 1998; Schroeder et al., 2003; Walsh and Stainier, 2001).

Although there is some debate on whether EMT is a prerequisite for the migration of AVC endocardial cells into the cardiac jelly during endocardial cushion formation in zebrafish, the mature AV valve of the 2-chambered zebrafish adult heart and 4-chambered mammalian valves are structurally similar. In addition, cellular and molecular signaling events occurring during heart valve development are largely conserved between zebrafish and mammals (Beis et al., 2005; Hu et al., 2000).

Cysteine-rich protein 2 (Crip2) is a Crip-type subgroup of the LIM domain protein family and has two conserved cysteine-rich motifs that contain cysteine and histidine residues with zincbinding properties (Karim et al., 1996; Tsui et al., 1996). Mouse Crip2 is reported to be highly expressed in the heart, brain, testis, kidney, and lung, and is involved in the dynamics of the cortical actin cytoskeleton by binding PTP-BL, a protein tyrosine phosphatase (van Ham et al., 2003). In zebrafish, Crip2 is reported to be a target of Wnt3a signaling and regulates smooth muscle cell differentiation (Kihara et al., 2011) and the development of cardiac neural crest cells during early embryogenesis (Sun et al., 2008). However, the direct role of Crip2 in the $A V$ valve formation has not yet been elucidated.

Zebrafish is a useful animal model for studying development of the heart and other circulation systems, including blood and lymphatic vessels (Kim and Kim, 2014, Kim et al., 2012a; 2013a; 2013b;) because the embryos are transparent and a multitude of transgenic lines that express fluorescent proteins in tissue- and cell-specific manners are readily available. In zebrafish, the heart can be observed under a dissecting microscope soon after formation of the primitive heart tube. The heart starts beating at $24 \mathrm{~h}$ post-fertilization (hpf), heart looping begins at 36 $\mathrm{hpf}$, functional valves are formed by $48 \mathrm{hpf}$, and heart valve development is complete by approximately $55 \mathrm{hpf}$ (Stainier et al., 2002).

In this study, we found that crip2 expression in the AVC endocardial cells, but not in the AVC myocardial cells, in late heart development is critical for heart valve development in zebrafish embryos. Heart looping was disrupted by crip2 knockdown. Furthermore, Crip2-deficient embryos showed markedly increased expression of the has 2 and versican a genes, which are responsible for the synthesis of Versican a and Hyaluronan. We provide data that Crip2 expressed in the AVC endothelial cells plays an important role in cardiac valve formation by suppressing genes involved in the synthesis of Versican a and Hyaluronan, which are essential ECM components in the cardiac jelly and endocardial cushion.

\section{MATERIALS AND METHODS}

Maintenance of zebrafish and collection of embryos

Zebrafish (Danio rerio) were raised and maintained at $28.5^{\circ} \mathrm{C}$, and embryos were staged as described in a previous study (Kimmel et al., 1995). Embryos were obtained from spontaneous spawnings and the embryos were manually dechorionated at appropriate stages by using watchmaker's forceps and then fixed using $4 \%$ paraformaldehyde in phosphate-buffered saline. Tg (cmlc2:EGFP) (Huang et al., 2003) and Tg (flk1:EGFP) (Jin et al., 2005) embryos were used for phenotypic analyses and immunostaining.
Whole-mount in situ hybridization and immunostaining Antisense digoxigenin-labeled RNA probes for crip2, vmhc, $c m l c 2$, cdh5, bmp4, tbx2b, notch1b, versican $a$, and has2 were generated using in vitro transcription with T3, T7, or SP6 RNA polymerase, according to the manufacturer's instructions (Roche, Germany). Whole-mount in situ hybridization was performed at the desired embryonic stages, as described by Jowett and Lettice (1994). For fluorescent immunostaining, a crip2 riboprobe was synthesized using fluorescein-12-UTP (Roche). After in situ hybridization, transgenic embryos were immunostained by anti-GFP antibody (Santa Cruz Biotechnology, Inc., USA). Processed embryos were photographed using a microscope (Axioskop, Zeiss, Germany) outfitted with a Nikon Coolpix 4500 digital camera system and a laser-scanning confocal microscope (LSM 5 PASCAL).

Antisense morpholino oligonucleotide (MO) and sense mRNA injections

crip2 antisense morpholino oligonucleotide (crip2 MO) was designed and ontained from Gene Tools LLC. (USA) to block the splicing of crip2 exon 2 and intron 2 and had the following sequence: 5'-CATGCCGAGGTGAGTAAAACAGAGA-3'. To generate crip2 sense mRNA, the full open reading frame of crip2 (NM_213497) was cloned into the pCS2+ expression vector. The construct was linearized using Notl digestion and capped RNA was synthesized using $\mathrm{m}^{7} \mathrm{G}\left(5^{\prime}\right)$ ppp $\left(5^{\prime}\right) \mathrm{G}$ (Roche) and SP6 RNA polymerase (Roche). The integrity of the synthetic RNA was examined on a $0.7 \%$ agarose gel. The RNA was diluted to $100 \mathrm{ng} / \mu \mathrm{l}$ and phenol red was added to a final concentration of $0.2 \%$ immediately before injection. The synthesized crip2 $\mathrm{MO}$ and sense mRNA were injected into oneor two-cell stage embryos by using a gas driven microinjector (World Precision Instruments, USA).

\section{RESULTS AND DISCUSSION}

In an in situ hybridization-based screen for candidate genes that regulate heart valve development, we found that crip2 expression was restricted to the AVC region in 48-hpf zebrafish embryos (Fig. 1 and data not shown). Detailed analysis of the gene expression profile showed that crip2 mRNA was detected in zebrafish embryos at the one-cell stage, indicating that crip2 was maternally expressed (Fig. 1A). At the shield stage, crip2 was ubiquitously expressed in the entire embryonic cell (Fig. $1 \mathrm{~B})$, but its expression became restricted to the mesodermal region along the anterior-posterior axis at the 10 somite-stage (ss) (Fig. 1C). In 18 ss embryos, crip2 expression became further restricted to the head and trunk mesoderm, including the somites, lateral plate mesoderm, and notochord posterior edge (Fig. 1D). In 24 hpf embryos, crip2 transcripts were abundant in the head, trunk, and tail (Fig. 1E). Transverse sections of the posterior trunk region further confirmed its expression in the somitic muscle (Fig. 1F) and blood vessels (Fig. 1G). At 42 hpf, crip2 expression had mostly disappeared from the head and trunk (Fig. $1 \mathrm{H})$, and new expression appeared in the pharyngeal arch blood vessels (Fig. 1I) and fin buds (Fig. 1J). These gene expression profiling data suggest a function of zygotic Crip2 in mesoderm-derived tissue formation during early embryogenesis.

During heart development, crip2 expression was observed in the linear heart tube in $24 \mathrm{hpf}$ embryos (Fig. 1K) and its strong expression persisted in the ventricle and $\mathrm{AV}$ canal regions at 36 hpf, but was barely detected in the atrium (Fig. 1L). In the looping heart tubes of 42 and 48 hpf embryos, crip2 expression was 


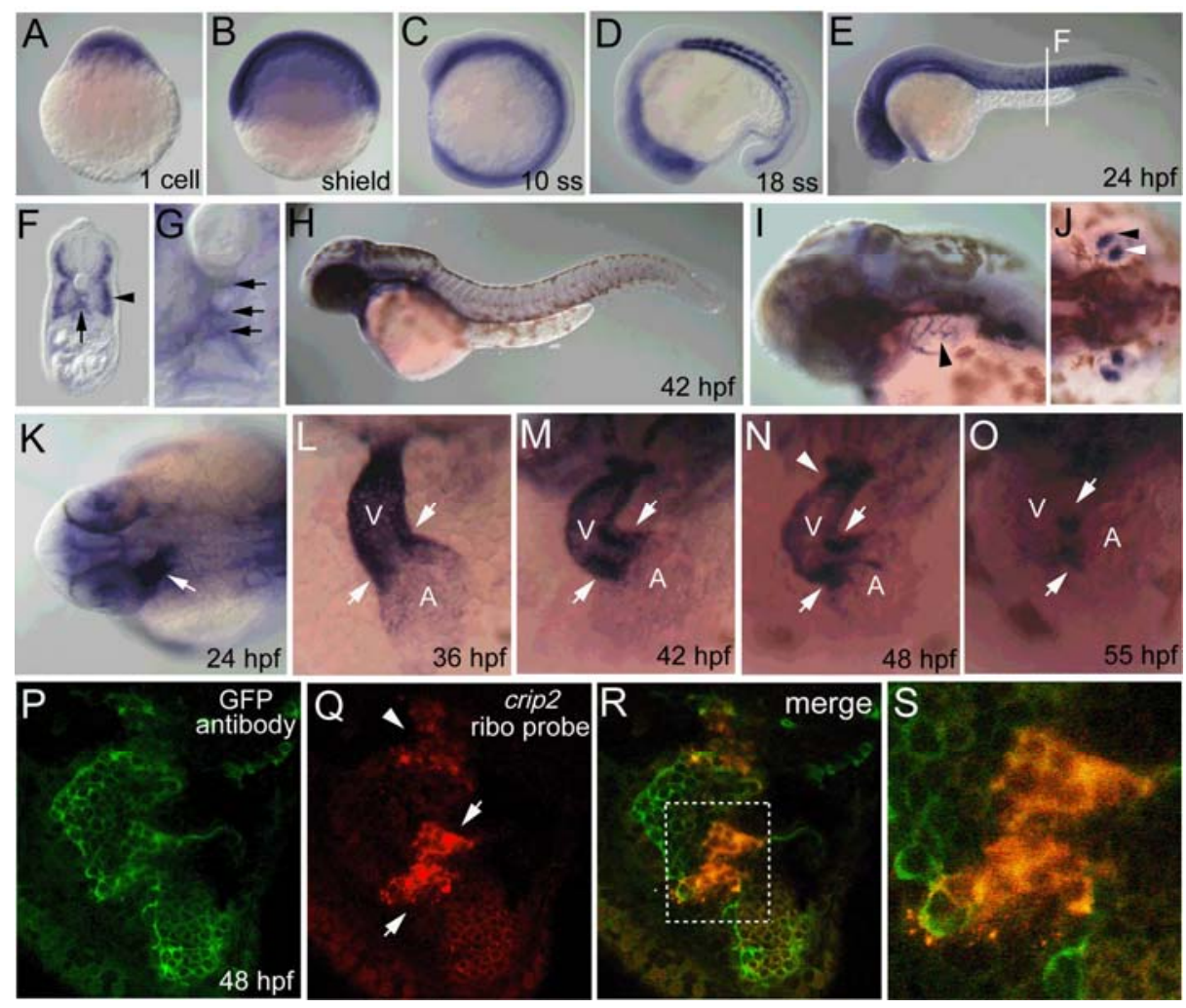

Fig. 1. Expression profile of crip2 in the developing zebrafish heart. (A-O) Embryos were stained using the crip2 riboprobe at the desired developmental stages. Lateral (A-E, H, and I), dorsal $(\mathrm{J}, \mathrm{K})$, and anterior views $(\mathrm{L}-\mathrm{O})$. Images of transverse sections of the trunk region at $24 \mathrm{hpf}(\mathrm{F}$, G). (A-J) crip2 expression during early zebrafish embryogenesis. Maternal expression at the one-cell stage (A) and ubiquitous expression at the shield stage (B). Expression of crip2 was detected in the entire mesodermal region at 10 ss (C). At 18 ss, crip2 expression became restricted to the head and trunk mesoderm region, including the somitic muscle, lateral plate mesoderm, and posterior notochord edge (D). At 24 hpf, crip2 was highly expressed in the whole embryonic body (E). Expression of crip2 in the somitic muscle (arrowhead) and blood vessels (arrow) in a transverse section of the trunk region at $24 \mathrm{hpf}(\mathrm{F})$. Enlarged view of crip2 expression in the blood vessels (arrows) in panel (F) (G). At $42 \mathrm{hpf}$, crip2 expression largely decreased in the whole embryonic body $(\mathrm{H})$. However, crip2 expression increased in the pharyngeal arch blood vessels [arrowhead in (I)] and fin buds [arrowheads in (J)]. (K-O) Expression of crip2 in the developing zebrafish heart. At $24 \mathrm{hpf}$, expression of crip2 was detected in the linear heart tube as indicated by an arrow (K). At 36 hpf, crip2 was highly expressed in the ventricle and AVC region (arrows), but it was nearly undetectable in the atrium (L). Expression of crip2 decreased in the ventricle and was restricted to the AVC region (arrows) and outflow tract (arrowhead) at $42(\mathrm{M})$ and $48 \mathrm{hpf}(\mathrm{N})$. At $55 \mathrm{hpf}(\mathrm{O})$, crip2 expression had mostly disappeared in the ventricle and atrium, but remained in the AVC region (arrows) (O). (P-Q) Laser-scanning confocal images showing fluorescent whole-mount in situ hybridization at $48 \mathrm{hpf}$. All embryos are shown in frontal view. All endocardial cells were marked using an anti-GFP antibody (green) in Tg(flk1:EGFP) embryos (P). Fluorescent crip2 riboprobe (red) was detected at the AVC (arrows) and outflow tract (arrowhead) (Q). Merged image of $P$ and $Q$ showed that only AVC endocardial cells (yellow color) are positive for both crip2 expression and GFP antibody (R). Enlarged image of the boxed region in $R(S)$. V, ventricle; $A$, atrium.

decreased in the ventricle and remained only at the AVC and outflow tract (Figs. $1 \mathrm{M}$ and $1 \mathrm{~N}$ ). When heart valve development was completed at $55 \mathrm{hpf}$, crip2 expression at the AVC and outflow tract had started to decrease (Fig. 10).

In $T g$ (flk1:EGFP) transgenic zebrafish embryos, green fluorescent protein (GFP) is expressed in all endothelial cells (Jin et al., 2005). To verify which cells express crip2, $48 \mathrm{hpf} T \mathrm{Tg}$ (flk1:EGFP) embryos were immunostained with GFP antibodies and then whole-mount in situ hybridization was performed using a fluorescent crip2 riboprobe. Endocardial cells in the ventricle, atrium, and AVC region were visualized using the GFP antibody (Fig. 1P), and expression of crip2 was detected in cells at the AVC and outflow tract (Fig. 1Q). By merging the images in Figs. $1 \mathrm{P}$ and $1 \mathrm{Q}$, we confirmed that crip2 is expressed specifically in the endocardium of the AVC and outflow tract and not in the heart chamber myocardium or endocardium during the late stage of heart development (Figs. 1R and 1S).

To evaluate the contribution of crip2 to late heart development, we designed a crip2 splice morpholino (crip2 MO) (Fig. 2A). The crip2 MO effectively blocked splicing between exon 2 and intron 2 and resulted in a decrease of crip2 mRNA transcript (Fig. 2B, ${ }^{* *}$ ) and an increase in the aberrant transcript containing intron 2 (Fig. 2B, *) in 24 hpf embryos. Similarly, the abundance of the crip2 mRNA transcripts was clearly reduced by the crip2 $\mathrm{MO}$ (Figs. 2D and 2D') in $24 \mathrm{hpf}$ embryos when compared to non-injection control embryos (NICs; Figs. 2C and $2 \mathrm{C}^{\prime}$ ) examined using whole-mount in situ hybridization.

To analyze the effect of crip2 knockdown on heart tube formation, we used $\mathrm{Tg}$ (cmlc2:EGFP) transgenic embryos that express GFP in myocardial cells in both ventricular and atrial 

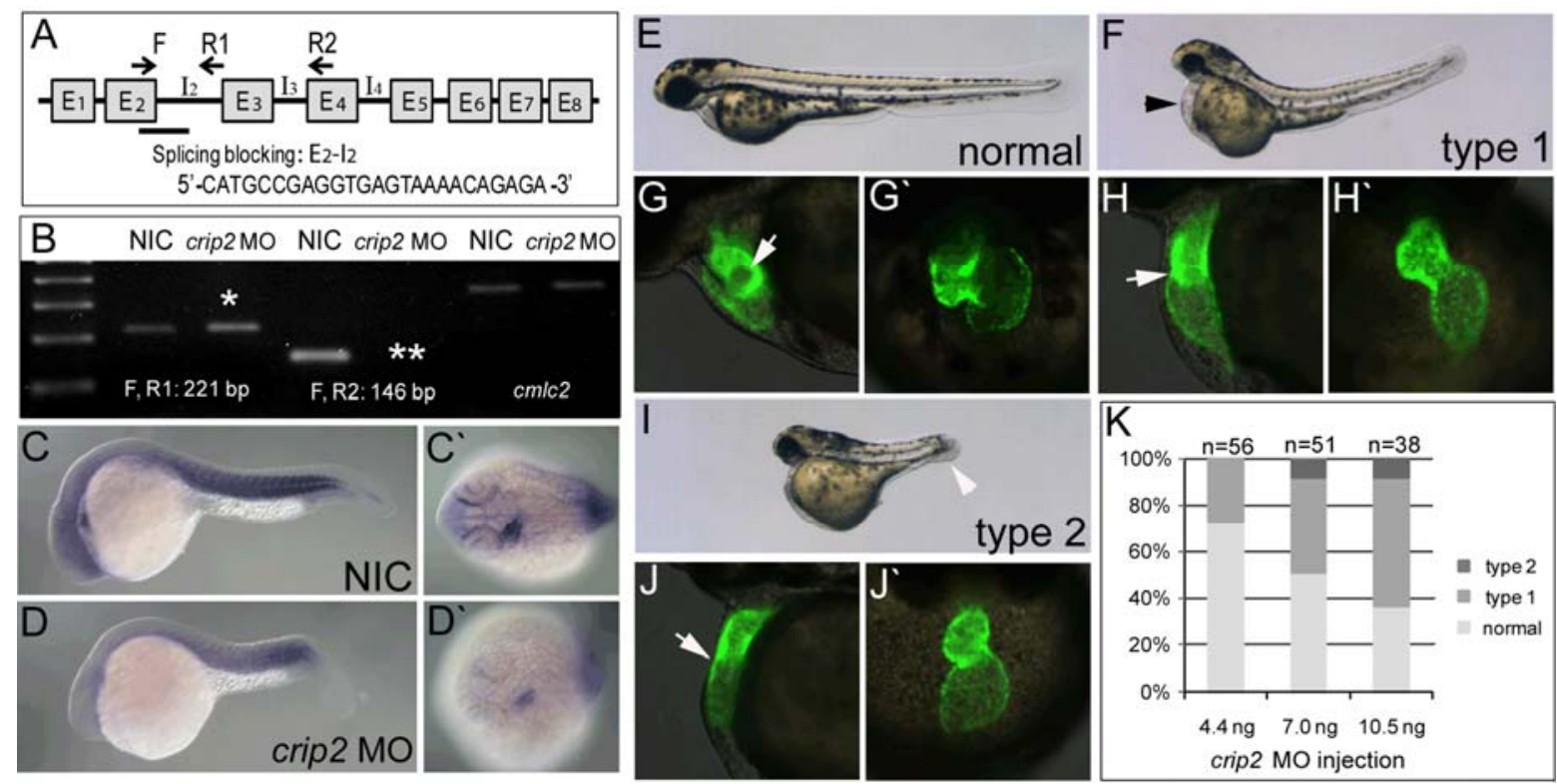

Fig. 2. Knockdown of crip2 induces a looping defect in the zebrafish embryonic heart. (A) A schematic diagram showing the designed crip2 morpholino oligonucleotide (crip2 MO) and RT-PCR primers (F, R1, and R2). (B-D') The in vivo activity of crip2 MO was evaluated using RTPCR (B). Impaired splicing products of crip2 mRNA $\left(^{*}\right)$ in the crip2 morphant embryos (crip2 MO) was amplified by RT-PCR using primer F/R1 $\left(^{*}\right)$ and primer F/R2 $\left(^{* *}\right)$ sets. Using the same primers, normally spliced mRNAs in the non-injection control (NIC) embryos were amplified by RT-PCR (221 bp, F/R1 set; 146 bp, F/R2 set). As a negative control, cmlc2 was amplified. (C-D') Effect of crip2 MO was confirmed by wholemount in situ hybridization using a crip2 riboprobe at $24 \mathrm{hpf}$. NIC (C, $\left.\mathrm{C}^{\prime}\right)$ and crip2 morphant embryos (D, D'). Lateral (C, D) and dorsal views $\left(C^{\prime}, D^{\prime}\right)$. (E-J') Phenotypic analysis of the Tg(cmlc2:EGFP) NIC and Tg(cmlc2:EGFP) crip2 morphant embryos. Type 1 morphant embryos showed cardiac edema (arrowhead) and a curved trunk (F). Bright-field (E, F, and I) and fluorescence microscopy images (G-H', J, and $\left.J^{\prime}\right)$. Lateral views (E-G, H, I, and $J$ ) and frontal views $\left(G^{\prime}, H^{\prime}\right.$, and $\left.J^{\prime}\right)$. The formation of a typical ring structure (arrow) in the AVC region (G) and $S-$ shaped looping in the NIC embryos $\left(\mathrm{G}^{\prime}\right)$ were hindered in crip2 morphant embryos $\left(\mathrm{H}, \mathrm{H}^{\prime}\right)$. Type 2 morphant embryos showed shortened trunks and tails (arrowhead) (I). Similar to type 1 morphants $\left(\mathrm{H}, \mathrm{H}^{\prime}\right)$, the formation of the AVC ring structure (arrows) $(\mathrm{J})$ and heart-looping process $\left(\mathrm{J}^{\prime}\right)$ were also disrupted in type 2 morphant embryos $\left(\mathrm{J}, \mathrm{J}^{\prime}\right)$. The dose-dependent effect of crip2 MO on the generation of the morphant phenotype (K).

portions of the heart tube (Huang et al., 2003). Depending on the amount of crip2 MO injected into one-cell stage embryos, three variable phenotypes were induced in $48 \mathrm{hpf}$ embryos: normal (Fig. 2E), type 1 (Fig. 2F), and type 2 phenotypes (Fig. 2I). In normal embryos, S-shaped heart looping (Fig. 2G') and typical ring structures in the AVC region (arrow) were observed (Fig. 2G). However, typical ring structures were not formed in the type 1 and type 2 embryos because heart tubes failed to undergo looping (Figs. $2 \mathrm{H}, 2 \mathrm{H}^{\prime}, 2 \mathrm{~J}$, and $2 \mathrm{~J}^{\prime}$ ). Because type 2 morphant embryos (Fig. 2l) showed more severe developmental defects than type 1 morphants, type 1 embryos were selected for further analysis of Crip2 function in heart development. The ratios of the dose-dependent phenotypes were calculated and these data are summarized in Fig. $2 \mathrm{~K}$. Collectively, our data demonstrating that morphological heart valve defects occur in crip2 morphant embryos and that crip2 expression becomes spatiotemporally restricted to the endocardium of the AVC and outflow tract strongly suggest an important role for Crip2 in heart valve development.

To further assess the function of Crip2 in late heart development, including heart valve formation, we analyzed the expression of cardiac marker genes (Fig. 3). During heart tube assembly, cardiac myosin light chain 2 (cmlc2) is expressed in myocardia throughout both ventricular and atrial portions of the heart tube, whereas ventricular myosin heavy chain ( $v m h c)$ is restricted to the myocardia in the ventricular portion (Yelon et al., 1999). VE-cadherin (cdh5), on the other hand, is specifically expressed in the endocardium during heart development (Larson et al., 2004). In zebrafish embryos, knockdown of crip2 did not alter the expression of $v m h c$ (Figs. 3A and 3B) and cm/c2 (Figs. 3C and 3D). The total abundance of $c d h 5$ mRNA was also not changed by crip2 $\mathrm{MO}$ (Figs. 3E and 3F), suggesting that Crip2 is not involved in the development of myocardium and endocardium comprising the outer layers of heart chambers.

Next, we examined the expression of genes required for the development of the heart valve. bmp4 is known to be highly expressed specifically in the AVC myocardium in zebrafish embryos (Larson et al., 2004). In the crip2 morphant embryos, bmp4 was expressed in the AVC region (Fig. $3 \mathrm{H}$, arrows), in a manner similar to that of the control embryos (Fig. 3G). In agreement with this, expression of $t b x 2 b$, a marker of the AVC myocardium (Walsh and Stainier, 2001), was also not altered (Figs. $3 \mathrm{l}$ and $3 \mathrm{~J}$, arrows). In mice, Notch promotes EMT during heart valve development and inhibition of Notch activity prevents valve formation in mice and zebrafish (Timmerman et al., 2004). notch $1 b$, a mouse notch 1 ortholog, was expressed in the AVC endocardium of $48 \mathrm{hpf}$ zebrafish embryos (Hurlstone et al., 2003), thus we tested the expression of notch $1 b$ in crip2 morphant embryos. We found that the expression level of notch $1 b$ detected in the AVC region of morphant embryos was 


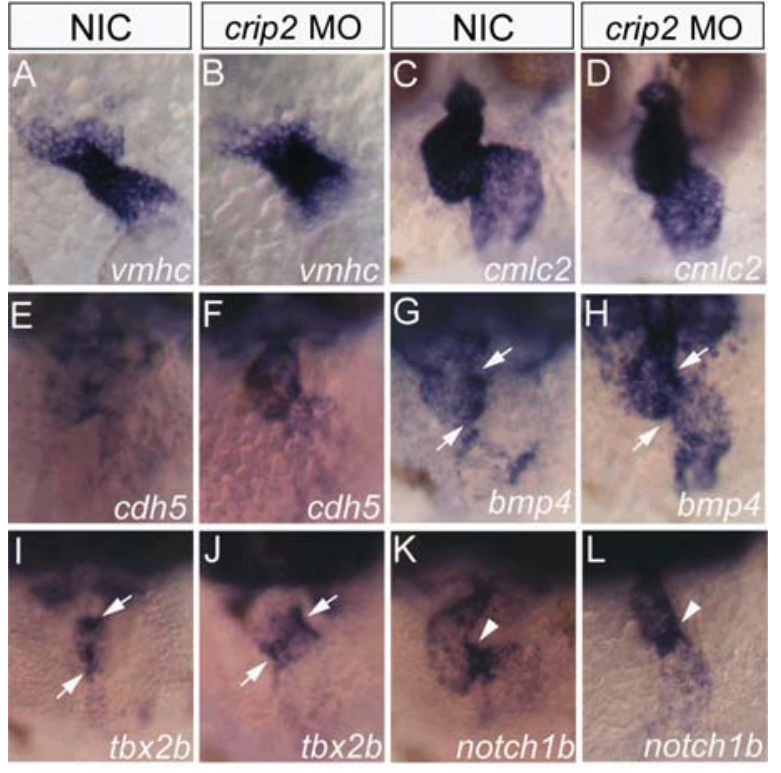

Fig. 3. Knockdown of crip2 does not affect the expression of myocardial and endocardial marker genes during heart development (A-L) Whole-mount in situ hybridization using vmhc (A, B), cmlc2 (C, $\mathrm{D}), \operatorname{cdh} 5(\mathrm{E}, \mathrm{F}), \operatorname{bmp} 4(\mathrm{G}, \mathrm{H}), t b x 2 b(\mathrm{I}, \mathrm{J})$, and ntoch1b (K, L) riboprobes in the NIC (A, C, E, G, I, and K) and crip2 morphant embryos (B, D, F, H, J, and $\mathrm{L})$ at $24 \mathrm{hpf}(\mathrm{A}, \mathrm{B})$ and $48 \mathrm{hpf}(\mathrm{C}-\mathrm{L})$. Dorsal views $(A, B)$ and frontal views $(C-L)$. Arrows indicate the AVC region.

comparable to that of control embryos at $48 \mathrm{hpf}$ (Figs. 3K and $3 \mathrm{~L})$. This observation indicated that Crip2 is not involved in regulating the EMT process in the AVC endocardium. An appropriate ECM environment in the endocardial cushion is reported to be required for normal heart valve formation (Camenisch et al., 2000; Schroeder et al., 2003). In zebrafish, it is well established that versican $a$ and has 2 synthesize versican a and hyaluronan, respectively, and that these genes have restricted expression in the AVC region in $48 \mathrm{hpf}$ embryos (Hurlstone et al., 2003). To further investigate whether Crip2 regulates synthesis of ECM components, we analyzed the expression of versican a and has2 in crip2 morphant embryos. The expression of versican a in the AVC region was significantly increased in the morphant embryos (Fig. 4B) compared to the control embryos (Fig. 4A). Similar to versican a, the expression of has2 in the AVC region was markedly upregulated by the knockdown of crip2 (Figs. 4C and 4D). These results imply that Crip2 functions as a negative regulator of ECM-related gene expression during $\mathrm{AV}$ valve development.

We also tested whether overexpression of crip2 could downregulate versican a and has2 expression. In wild-type embryos, overexpression of crip2 did not decrease the expression of versican $a$ and has 2 , although severe developmental defects, including curled body and cardiac edema, were observed (Supplementary Fig. 1). These results suggested that the expression level of Crip2 in wild-type embryos is sufficient to suppress the expression of versican a and has 2; thus, further suppression of these ECM genes could not be achieved by the overexpression of crip2. Alternatively, Crip2 might require a cofactor to suppress versican $a$ and has 2 expression. There

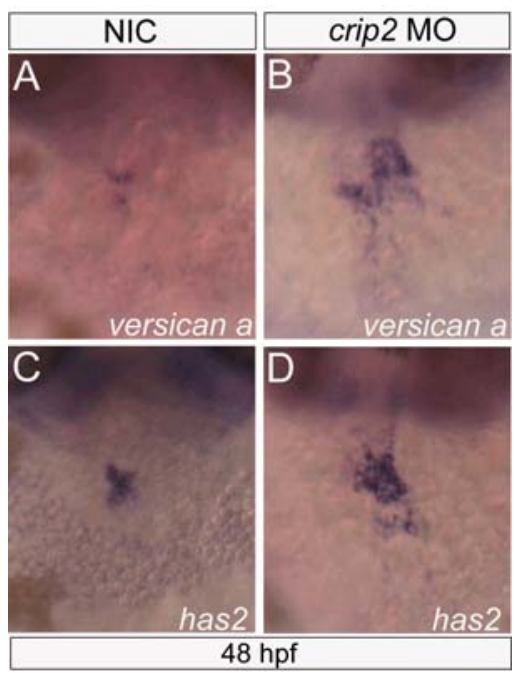

Fig. 4. Increased expression of versican a and has2 in crip2 morphant embryos. (A-D) Whole-mount in situ hybridization data showing expression of versican a $(\mathrm{A}, \mathrm{B})$ and has2 $(\mathrm{C}, \mathrm{D})$ in the AVC region of $48 \mathrm{hpf}$ embryos. Frontal views of the NIC (A, C) and crip2 morphant embryos $(B, D)$.

-fore, overexpression of crip2 alone cannot further suppress the expression of versican a and has 2 .

Adenomatous polyposis coli (APC), a well-known tumor suppressor protein, negatively regulates $\mathrm{Wnt} / \beta$-catenin signaling (Hurlstone et al., 2003). Wnt/ $\beta$-catenin signaling can be activated by APC inactivation in mutant mice. Moreover, heart valve defects in APC null mice are reported to be caused by increased versican a and has2 expression in the AVC region, indicating that versican a and has2 are transcriptional targets of the Wnt/ $\beta$-catenin pathway (Hurlstone et al., 2003). According to our crip2 knockdown data, expression of versican a and has2 at the AVC was markedly upregulated, suggesting an antagonistic role of Crip2 towards Wnt/B-catenin signaling that activates versican a and has2 expression. Our results, together with that of previous reports, indicate that either deficiency or over-production of Versican and Has2 causes heart valve defects (Hurlstone et al., 2003; Schroeder et al., 2003; Walsh and Stainier, 2001).

Here, we report a novel function of Crip2 in suppressing the expression of versican a and has2 in the AVC region, which is essential for normal heart valve development. Through its spatiotemporal regulation of gene expression, Crip2 likely contributes to the formation of an appropriate ECM environment in the endocardial cushion region during multiple stages of heart valve development.

Note: Supplementary information is available on the Molecules and Cells website (www.molcells.org).

\section{ACKNOWLEDGMENTS}

We thank the Korea Zebrafish Organogenesis Mutant Bank (ZOMB) and Zebrafish Information Network (ZFIN) for providing the zebrafish lines. This work was supported by the Kyungpook National University Research Fund (2012) and the Basic Science Research Program through the National Research Foundation of Korea (NRF), funded by the Ministry of Education (NRF-2013R1A1A2009570). 


\section{REFERENCES}

Armstrong, E.J., and Bischoff, J. (2004). Heart valve development endothelial cell signaling and differentiation. Circ. Res. 95, 459470.

Beis, D., Bartman, T., Jin, S.W., Scott, I.C., D'Amico, L.A., Ober, E.A., Verkade, H., Frantsve, J., Field, H.A., Wehman, A., et al. (2005). Genetic and cellular analyses of zebrafish atrioventricular cushion and valve development. Development 132, 41934204.

Camenisch, T.D., Spicer, A.P., Brehm-Gibson, T., Biesterfeldt, J., Augustine, M.L., Calabro, A., Kubalak, S., Klewer, S.E., and McDonald, J.A. (2000). Disruption of hyaluronan synthase-2 abrogates normal cardiac morphogenesis and hyaluronanmediated transformation of epithelium to mesenchyme. J. Clin. Invest. 106, 349-360.

Combs, M.D., and Yutzey, K.E. (2009). Heart valve development: regulatory networks in development and disease. Circ. Res. 105, 408-421.

Délot, E.C. (2003). Control of endocardial cushion and cardiac valve maturation by BMP signaling pathways. Mol. Genet. Metab. 80, 27-35.

Hu, N., Sedmera, D., Yost, H.J., and Clark, E.B. (2000). Structure and function of the developing zebrafish heart. Anat. Rec. 260, 148-157.

Huang, C.J., Tu, C.T., Hsiao, C.D., Hsieh, F.J., and Tsai, H.J. (2003). Germ-line transmission of a myocardium-specific GFP transgene reveals critical regulatory elements in the cardiac myosin light chain 2 promoter of zebrafish. Dev. Dyn. 228, 30-40.

Hurlstone, A.F., Haramis, A.P., Wienholds, E., Begthel, H., Korving, J., Van Eeden, F., Cuppen, E., Zivkovic, D., Plasterk, R.H., and Clevers, H. (2003). The Wnt/beta-catenin pathway regulates cardiac valve formation. Nature 425, 633-637.

Jiao, K., Kulessa, H., Tompkins, K., Zhou, Y., Batts, L., Baldwin, H.S., and Hogan, B.L. (2003) An essential role of Bmp4 in the atrioventricular septation of the mouse heart. Genes Dev. 17, 2362-2367.

Jin, S.W., Beis, D., Mitchell, T., Chen, J.N., and Stainier, D.Y (2005). Cellular and molecular analyses of vascular tube and lumen formation in zebrafish. Development 132, 5199-5209.

Jowett, T., and Lettice, L. (1994). Whole-mount in situ hybridizations on zebrafish embryos using a mixture of digoxigenin- and fluorescein-labelled probes. Trends Genet. 10, 73-74.

Karim, M.A., Ohta, K., Egashira, M., Jinno, Y., Niikawa, N., Matsuda, I., and Indo, Y. (1996). Human ESP1/CRP2, a member of the LIM domain protein family: characterization of the cDNA and assignment of the gene locus to chromosome 14q32.3. Genomics 31, 167-176.

Kihara, T., Shinohara, S., Fujikawa, R., Sugimoto, Y., Murata, M., and Miyake, J. (2011). Regulation of cysteine-rich protein 2 localization by the development of actin fibers during smooth muscle cell differentiation. Biochem. Biophys. Res. Commun. 411, 96101.

Kim, J.D., and Kim, J. (2014). Alk3/Alk3b and Smad5 Mediate BMP signaling during lymphatic development in zebrafish. Mol. Cells $37,270-274$

Kim, J.D., Kang, H., Larrivée, B., Lee, M.Y., Mettlen, M., Schmid S.L., Roman, B.L., Qyang, Y., Eichmann, A., and Jin, S.W. (2012a). Context-dependent proangiogenic function of bone morphogenetic protein signaling is mediated by disabled homolog 2. Dev. Cell 23, 441-448.

Kim, Y.S., Kim, M.J., Koo, T.H., Kim, J.D., Koun, S., Ham, H.J., Lee, Y.M., Rhee, M., Yeo, S.Y., and Huh, T.L. (2012b). Histone deacetylase is required for the activation of $\mathrm{Wnt} / \beta$-catenin signaling crucial for heart valve formation in zebrafish embryos. Biochem. Biophys. Res. Commun. 423, 140-146.

Kim, J.D., Kang, Y., Kim, J., Papangeli, I., Kang, H., Wu, J., Park H., Nadelmann, E., Rockson, S.G., Chun, H.J., et al. (2013a) Essential role of Apelin signaling during lymphatic development in zebrafish. Arterioscler. Thromb. Vasc. Biol. 34, 338-345.

Kim, S.H., Schmitt, C.E., Woolls, M.J., Holland, M.B., Kim, J.D., and Jin, S.W. (2013b). Vascular endothelial growth factor signaling regulates the segregation of artery and vein via ERK activity during vascular development. Biochem. Biophys. Res. Commun. 430, 1212-1216.

Kimmel, C.B., Ballard, W.W., Kimmel, S.R., Ullmann, B., and Schilling, T.F. (1995). Stages of embryonic development of the zebrafish. Dev. Dyn. 203, 253-310.

Krug, E.L., Runyan, R.B., and Markwald, R.R. (1985). Protein extracts from early embryonic hearts initiate cardiac endothelial cytodifferentiation. Dev. Biol. 112, 414-426.

Larson, J.D., Wadman, S.A., Chen, E., Kerley, L., Clark, K.J., Eide, M., Lippert, S., Nasevicius, A., Ekker, S.C., Hackett, P.B., et al. (2004). Expression of VE-cadherin in zebrafish embryos: a new tool to evaluate vascular development. Dev. Dyn. 231, 204-213.

Ma, L., Lu, M.F., Schwartz, R.J., and Martin, J.F. (2005). Bmp2 is essential for cardiac cushion epithelial-mesenchymal transition and myocardial patterning. Development 132, 5601-5611.

Mjaatvedt, C.H., Yamamura, H., Capehart, A.A., Turner, D., and Markwald, R.R. (1998). The Cspg2 gene, disrupted in the hdf mutant, is required for right cardiac chamber and endocardia cushion formation. Dev. Biol. 202, 56-66.

Nakajima, Y., Yamagishi, T., Hokari, S., and Nakamura, H. (2000) Mechanisms involved in valvuloseptal endocardial cushion formation in early cardiogenesis: roles of transforming growth factor (TGF)-beta and bone morphogenetic protein (BMP). Anat Rec. 258, 119-127.

Schroeder, J.A., Jackson, L.F., Lee, D.C., and Camenisch, T.D. (2003). Form and function of developing heart valves: coordination by extracellular matrix and growth factor signaling. J. Mol. Med. 81, 392-403.

Stainier, D.Y., Beis, D., Jungblut, B., and Bartman, T. (2002) Endocardial cushion formation in zebrafish. Cold Spring Harb. Symp. Quant. Biol. 67, 49-56.

Sun, X., Zhang, R., Lin, X., and Xu, X. (2008) Wnt3a regulates the development of cardiac neural crest cells by modulating expression of cysteine-rich intestinal protein 2 in rhombomere 6 . Circ. Res. 102, 831-839.

Timmerman, L.A., Grego-Bessa, J., Raya, A., Bertrán, E., PérezPomares, J.M., Díez, J., Aranda, S., Palomo, S., McCormick, F Izpisúa-Belmonte, J.C., et al. (2004) Notch promotes epithelialmesenchymal transition during cardiac development and oncogenic transformation. Genes Dev. 18, 99-115.

Tsui, S.K., Chan, P.P., Cheuk, C.W., Liew, C.C., Waye, M.M., Fung, K.P., and Lee, C.Y. (1996) A novel cDNA encoding for a LIM domain protein located at human chromosome $14 q 32$ as a candidate for leukemic translocation. Biochem. Mol. Biol. Int. 39, 747-754.

van Ham, M., Croes, H., Schepens, J., Fransen, J., Wieringa, B., and Hendriks, W. (2003) Cloning and characterization of mCRIP2, a mouse LIM-only protein that interacts with PDZ domain IV of PTP-BL. Genes Cells 8, 631-644.

Walsh, E.C., and Stainier, D.Y. (2001) UDP-glucose dehydrogenase required for cardiac valve formation in zebrafish. Science 293, 1670-1673

Yelon, D., Horne, S.A., and Stainier, D.Y. (1999). Restricted expression of cardiac myosin genes reveals regulated aspects of heart tube assembly in zebrafish. Dev. Biol. 214, 23-37. 